\title{
Pairwise quantum and classical correlations in multi-qubits states via linear relative entropy
}

\author{
M. Daoud ${ }^{a, b, 41}$, R. Ahl Laamara ${ }^{d, e} 2$ and H. El Hadfi ${ }^{d} 3$ \\ ${ }^{a}$ Max Planck Institute for the Physics of Complex Systems, Dresden, Germany \\ ${ }^{b}$ Abdus Salam International Centre for Theoretical Physics, Trieste, Italy \\ ${ }^{c}$ Department of Physics, Faculty of Sciences, University Ibnou Zohr, Agadir, Morocco \\ ${ }^{d}$ LPHE-Modeling and Simulation, Faculty of Sciences, Rabat, Morocco \\ ${ }^{e}$ Centre of Physics and Mathematics, Rabat, Morocco
}

\begin{abstract}
The pairwise correlations in a multi-qubit state are quantified through a linear variant of relative entropy. In particular, we derive the explicit expressions of total, quantum and classical bipartite correlations. Two different bi-partioning schemes are considered. We discuss the derivation of closest product, quantum-classical and quantum-classical product states. We also investigate the additivity relation between the various pairwise correlations existing in pure and mixed states. As illustration, some special cases are examined.
\end{abstract}

\footnotetext{
1 email: m_daoud@hotmail.com

2 email: ahllaamara@gmail.com

3 email: hanane.elhadfi@gmail.com
} 


\section{Introduction}

It is commonly accepted that the concept of quantum correlations plays a central role in quantum information processing [1, 2]. In this context, it is now well established that entanglement constitutes a valuable resource for many quantum and computational protocols, 3, 4, This explains the efforts dedicated to the development of a general theory of quantum, classical and total correlations in composite quantum systems. Various measures and several methods were reported in the literature (for a recent review see [5]). Entropic based measures, such as entanglement of formation, linear entropy and quantum discord have attracted considerable attention in the last decade [6, 7, 8, 9, 10, 11. Nowadays, the quantum correlations as a special feature of quantum systems continue to be investigated from various perspectives and for different purposes. Recently, it has become evident that the methods developed in quantum information will lead to further insight in various areas of physics. One may quote for instance quantum phase transitions in complex systems [5].

However, despite the information meaning of entropic measures, the explicit determination of entropy based correlations (like quantum discord) requires complex optimization procedures, even for the simplest case of two qubit systems. To overcome such difficulties, it was natural to look for other measures leading to more tractable and computable expressions of the information contained in a multipartite quantum system. Hence, as quantum states are represented by density matrices, geometric measures were used to evaluate the information of one system about another. In this scenario, using the Hilbert-Schmidt distance, a geometrized variant of quantum discord was proposed in [12. In addition, other geometrical approaches to quantify classical and quantum correlations in quantum systems were discussed in the literature [13, 14] (see also [15, 16, 17, 18]).

Another important issue in characterizing the nature of quantum correlations in a composite system concerns the classification of the total correlations in quantum, quantum-classical and classical parts and therefore the relationship among them. The first attempt towards a unified view of correlations in multipartite systems was developed in [19] using the notion of relative entropy. In the same vein, a geometric formulation based on Hilbert-Schmidt norm was reported in [13, 14].

The understanding of the correlation in many body systems, especially highly correlated ones, remains challenging from experimental as well as theoretical point of views. To quantify the overall amount of correlations in a multipartite system, it is natural to consider pairwise correlations. In this picture, the pairwise quantum correlations in $n$ qubit systems, possessing exchange and parity symmetries, were considered in [20, 21]. The original definition of quantum discord [22, 23] and its geometric version (geometric quantum discord) [12] were used to derive the explicit expressions of pairwise correlations in the system. However, in the mentioned papers (by two authors of us), very little attention has been paid to the classification of the states according to their degrees of quantum-

ness. This issue is especially important if one wants to construct a unified framework establishing the relations among all correlations in a multi-partite system, namely classical, quantum and total correlations. In this respect, we shall consider the linear relative entropy to evaluate the distance 
between a given state and its closest one without the desired property. We develop a quantitative unified scheme for bipartite correlations in a multi-qubit system. On the technical front, the linear relative entropy provides a significantly simple formalism to derive computable analytical expressions of bipartite correlations. This is due essentially to the simplifications of the optimization procedures which are intractable in the relative entropy based framework discussed in [19]. In the other hand, we show that the quantum discord based on linear relative entropy is equivalent to the pairwise geometric discord (using Hilbert-Schmidt trace) derived in [20] . We establish, via the linear relative entropy, a closed additivity relation between the various kinds of bipartite (total, quantum and classical) correlations. This is the second merit of the linear relative entropy in comparison with the geometric unified view reported in [13, 14] in which the bipartite correlations, as measured by Hilbert-Schmidt distance, violate the additivity relation found in [19]. We also derive the explicit forms of products states, the classically correlated states and the corresponding closest product states. This work completes further the analysis developed in [20, 21] concerning the characterization and the quantification of the various correlations existing in $n$-qubit systems with parity and exchange invariance.

This paper is organized as follows. In the first section, we introduce a specific multi-qubit state for which we shall study the pairwise correlations (total, quantum and classical) using the linear relative entropy. We particularly focus on $n$ qubit state possessing parity and exchange symmetries. Two different bi-partitions (pure and mixed) are considered. In section 3, the notion of linear relative entropy and its relationship with Hilbert-Schmidt norm are presented. A closed relation involving the various kind of correlations in a bipartite state is discussed. This relation can be viewed as a linear variant of the additivity relation introduced in [19] to deal with the different correlations present in a multipartite system in a common framework. In sections 4 and 5 , the expressions of all pairwise correlations present in the multi-qubit system under consideration are explicitly derived (section 4 concerns the pure bipartition scheme and section 5 is devoted to mixed states). Also, the expressions of the closest product, quantum-classical and quantum-classical product states are obtained. To

illustrate our results, some special cases are considered in section 6. Concluding remarks close this paper.

\section{Bipartite states in multi-qubit system and linear relative entropy}

Different methods were proposed in the literature to tackle the issue concerning the quantification of the correlations in multi-partite systems. For instance the Rulli-Sarandy approach [24] defines the global correlation, in a system comprising many parts, as the maximum of correlation existing among all possible bi-partitions. Another approach, by Z-H Ma and coworkers [25, quantifies the global correlation as the sum of all possible bi-partite correlations. Both definitions are based on the bipartite measures of the correlations in the system. In this paper, to investigate the pairwise correlation in a $n$ qubit system prepared in a symmetric pure state, we follow the procedure developed 
in [20]. The Hilbert space of this system is

$$
\mathcal{H}=\mathcal{H}_{1} \otimes \mathcal{H}_{2} \otimes \cdots \otimes \mathcal{H}_{n}
$$

where $\mathcal{H}_{i}(i=1,2, \cdots, n)$ is spanned by the vectors $|0\rangle$ and $|1\rangle$. An arbitrary single qubit writes

$$
|\omega, \phi\rangle=\sqrt{\omega}|0\rangle+\sqrt{1-\omega} e^{i \phi}|1\rangle
$$

with $0 \leq \omega \leq 1$ and $\phi \in \mathbb{R}$. In this work, we shall focus on the following equally weighted or balanced multi-partite state

$$
|\omega, n\rangle=\mathcal{N}(\mid n, \omega,+)+\mid n, \omega,-)),
$$

where the states $\mid n, \omega, \pm)$ are given by

$$
\mid n, \omega, \pm)=|\omega, \pm\rangle_{1} \otimes|\omega, \pm\rangle_{2} \otimes \cdots \otimes|\omega, \pm\rangle_{n}
$$

with

$$
|\omega,+\rangle \equiv|\omega, 0\rangle \quad|\omega,-\rangle \equiv|\omega, \pi\rangle .
$$

In equation (2), the normalization factor $\mathcal{N}$ is

$$
\mathcal{N}=\left[2+2 s^{n}\right]^{-1 / 2}
$$

where $s$ denotes the scalar product between the states $|\omega,+\rangle$ and $|\omega,-\rangle$. It is defined by

$$
s=2 \omega-1 .
$$

Hereafter, we consider only states with $\frac{1}{2} \leq \omega \leq 1$ such that $0 \leq s \leq 1$. The situation where $0 \leq \omega \leq \frac{1}{2}$ can be deduced by replacing the state $|\omega, \phi\rangle$ by $|1-\omega, \phi\rangle$. The state $|\omega, \phi\rangle$ can be identified with the spin- $\frac{1}{2}$ coherent state with $|0\rangle \equiv\left|\frac{1}{2},-\frac{1}{2}\right\rangle$ and $|1\rangle \equiv\left|\frac{1}{2},+\frac{1}{2}\right\rangle$. Henceforth, the state (3) can be identified with a spin $j=n / 2$ coherent state [21]. A spin- $j$ coherent state is the tensor product of $2 j$ spin $\frac{1}{2}$ coherent states. In this respect, a pure separable state of $n=2 j$ qubits is necessary a spin- $j$ coherent state. Also, it is interesting to note that the states (2) can be viewed as the even spin coherent states [21.

Along the line of reasoning adopted in [20, two different kinds of bi-partitions of the $n$ qubit system (2) are possible. In the first scheme, the system is partitioned into two distinct subsystems. In the second scheme, a two-qubit state is obtained by tracing out $(n-2)$ qubit from the whole system. These two partitioning schemes are discussed in what follows. 


\subsection{Pairwise partitioning and qubit mapping}

\subsubsection{Bipartite pure states}

In the first scheme, the system is divided into two components. The first one contains $k$ qubits $(1 \leq k \leq n-1)$ and the second subsystem comprises the remaining $(n-k)$ qubits. In this setting, the state (2) reads as

$$
|\omega, n\rangle=\mathcal{N}\left(|\omega,+\rangle_{k} \otimes|\omega,+\rangle_{n-k}+|\omega,-\rangle_{k} \otimes|\omega,-\rangle_{n-k}\right),
$$

where

$$
\begin{gathered}
|\omega, \pm\rangle_{k}=|\omega, \pm\rangle_{1} \otimes|\omega, \pm\rangle_{2} \otimes \cdots \otimes|\omega, \pm\rangle_{k}, \\
|\omega, \pm\rangle_{n-k}=|\omega, \pm\rangle_{k+1} \otimes|\omega, \pm\rangle_{k+2} \otimes \cdots \otimes|\omega, \pm\rangle_{n} .
\end{gathered}
$$

In order to evaluate the information of one subsystem about another, we introduce a pair of two logical qubits by means of the orthogonal basis $\left\{|0\rangle_{k},|1\rangle_{k}\right\}$ defined as

$$
|0\rangle_{k}=\frac{|\omega,+\rangle_{k}+|\omega,-\rangle_{k}}{\sqrt{2\left(1+s^{k}\right)}}, \quad|1\rangle_{k}=\frac{|\omega,+\rangle_{k}-|\omega,-\rangle_{k}}{\sqrt{2\left(1-s^{k}\right)}}
$$

for the first sub-system . Similarly, for the second subsystem, we introduce the following two logical qubits $\left\{|0\rangle_{n-k},|1\rangle_{n-k}\right\}$ as

$$
|0\rangle_{n-k}=\frac{|\omega,+\rangle_{n-k}+|\omega,-\rangle_{n-k}}{\sqrt{2\left(1+s^{n-k}\right)}}, \quad|1\rangle_{n-k}=\frac{|\omega,+\rangle_{n-k}-|\omega,-\rangle_{n-k}}{\left.\sqrt{2\left(1-s^{n-k}\right.}\right)} .
$$

Inserting the definitions (6) and (7) in the expression of the multi-qubits state partitioned as in (5) and using the Schmidt decomposition, we obtain

$$
|\omega, n\rangle=\sqrt{\lambda_{+}}|\mathbf{0}\rangle_{k} \otimes|\mathbf{0}\rangle_{n-k}+\sqrt{\lambda_{-}}|\mathbf{1}\rangle_{k} \otimes|\mathbf{1}\rangle_{n-k},
$$

where the quantities given by

$$
\lambda_{ \pm}=\frac{1}{2}\left(1 \pm \frac{s^{k}+s^{n-k}}{1+s^{n}}\right)
$$

are the eigenvalues of the marginal density $\rho_{k}\left(\rho_{k}=\operatorname{Tr}_{(n-k)} \rho_{k, n-k}\right.$ with $\left.\rho_{k, n-k}=|\omega, n\rangle\langle\omega, n|\right)$ associated with the subsystem grouping $k$ qubits. In the Schmidt decomposition (8), the vectors $|\mathbf{0}\rangle_{k}$ and $|\mathbf{1}\rangle_{k}$ (resp. $|\mathbf{0}\rangle_{n-k}$ and $|\mathbf{1}\rangle_{n-k}$ ) are the eigenvectors of the marginal density $\rho_{k}$ (resp. $\rho_{n-k}=\operatorname{Tr}_{(k)} \rho_{k, n-k}$ ). The Fano-Bloch representation of the state (8) reads

$$
\rho_{k, n-k}=\frac{1}{4}\left[\sigma_{0} \otimes \sigma_{0}+R_{30} \sigma_{3} \otimes \sigma_{0}+R_{03} \sigma_{0} \otimes \sigma_{3}+\sum_{i=1}^{3} R_{i i} \sigma_{i} \otimes \sigma_{i}\right],
$$

where the correlation matrix elements are given by

$$
R_{30}=R_{03}=\lambda_{+}-\lambda_{-}, \quad R_{11}=-R_{22}=2 \sqrt{\lambda_{+} \lambda_{-}}, \quad R_{33}=1 .
$$

They rewrite explicitly as

$$
R_{30}=R_{03}=\frac{s^{k}+s^{n-k}}{1+s^{n}}, \quad R_{11}=-R_{22}=\frac{\sqrt{\left(1-s^{2 k}\right)\left(1-s^{2(n-k)}\right)}}{1+s^{n}}, \quad R_{33}=1
$$

in term of the overlapping factor $s$. 


\subsubsection{Bipartite mixed states}

The second procedure to extract two qubit states from the entire $n$ qubit system consists in tracing out all qubits except two. In this way, one obtains $n(n-1) / 2$ identical density matrices and the reduced density matrix describing a two qubit subsystem writes

$$
\begin{array}{r}
\rho_{12}=\mathcal{N}^{2}(|\omega,+, \omega,+\rangle\langle\omega,+, \omega,+|+| \omega,-, \omega,-\rangle\langle\omega,-, \omega,-| \\
\left.+s^{n-2}|\omega,-, \omega,-\rangle\left\langle\omega,+, \omega,+\left|+s^{n-2}\right| \omega,+, \omega,+\right\rangle\langle\omega,-, \omega,-|\right) .
\end{array}
$$

It can be also expressed as a two rank operator

$$
\rho_{12}=\frac{1}{2}\left(1+s^{n-2}\right) \frac{\mathcal{N}^{2}}{\mathcal{N}_{+}^{2}}\left|\omega_{+}\right\rangle\left\langle\omega_{+}\left|+\frac{1}{2}\left(1-s^{n-2}\right) \frac{\mathcal{N}^{2}}{\mathcal{N}_{-}^{2}}\right| \omega_{-}\right\rangle\left\langle\omega_{-}\right|,
$$

where

$$
\left|\omega_{ \pm}\right\rangle=\mathcal{N}_{ \pm}(|\omega,+\rangle \otimes|\omega,+\rangle \pm|\omega,-\rangle \otimes|\omega,-\rangle)
$$

and

$$
\mathcal{N}_{ \pm}^{2}=2 \pm 2 s^{2}
$$

In the Fano-Bloch representation, the state $\rho_{12}(14)$ takes the form

$$
\rho_{12}=\sum_{\alpha \beta} \mathcal{R}_{\alpha \beta} \sigma_{\alpha} \otimes \sigma_{\beta},
$$

where the non-vanishing matrix elements $\mathcal{R}_{\alpha \beta}(\alpha, \beta=0,1,2,3)$ are

$\mathcal{R}_{00}=1, \quad \mathcal{R}_{11}=2 \mathcal{N}^{2}\left(1-s^{2}\right), \quad \mathcal{R}_{22}=-2 \mathcal{N}^{2}\left(1-s^{2}\right) s^{n-2}, \mathcal{R}_{33}=2 \mathcal{N}^{2}\left(s^{2}+s^{n-2}\right), \quad \mathcal{R}_{03}=\mathcal{R}_{30}=2 \mathcal{N}^{2}\left(s+s^{n-1}\right)$

\section{Pairwise correlations using linear relative entropy}

In the unified view presented in [19], the relative entropy based measures of quantum, classical and total correlations satisfy a closed additivity relation. Unfortunately, this approach requires minimization procedures that are in general impossible to achieve analytically. In this section, we present an alternative description to overcome these analytical problems. In this order, exploiting the pairwise qubit mapping discussed in the previous section (see also [20]) and the concept of linear relative entropy, we propose a unified scheme to deal equally with the various kinds of correlations in a bipartite system. Hence, the main objective of this section is to develop a suitable quantification of the pairwise correlations present in the $n$-qubit state (2) using the concept of linear relative entropy. This provides us with a simple way to derive the explicit expressions of the different pairwise correlations (classical, quantum and total) in the bipartite states $\rho_{k, n-k}$ (10) and $\rho_{12}$ (15). 


\subsection{Correlation quantifiers based on relative entropy}

To begin, we first review the essential of the unifying view of correlations developed in [19] based on the formalism of the relative entropy defined by

$$
S(\rho \| \sigma)=-\operatorname{Tr}(\rho \log \sigma)-S(\rho),
$$

where $S(\rho)=-\operatorname{Tr}(\rho \log \rho)$ is the von Neumann entropy. It constitutes an adequate tool to decide about the dissimilarity between two quantum states. Using this measure Modi et al [19] have shown that the closest product state $\pi_{\rho}$ from any density operator $\rho$ is given by the product of the marginal matrices and the total correlation is given by the differences of von Neumann entropies as

$$
T=S\left(\rho \| \pi_{\rho}\right)=S\left(\pi_{\rho}\right)-S(\rho)
$$

Similarly, quantum correlation can be viewed as a minimum loss due to measurements in the sense of quantum mutual information. The information in a classically correlated state is not disturbed by local measurements. This state is called a quantum-classical state (sometimes also referred to as classical state [19]) and it is of the form

$$
\chi_{\rho}=\sum_{i, j} p_{i, j}|i\rangle\langle i|\otimes| j\rangle\langle j|,
$$

where $p_{i, j}$ are the probabilities with $\{|i\rangle,|j\rangle\}$ local basis. The quantum correlation is then defined as the minimal distance between the state $\rho$ and the quantum-classical states $\chi_{\rho}$. It is given by the differences between von Neumann entropies as

$$
D=S\left(\rho \| \chi_{\rho}\right)=S\left(\chi_{\rho}\right)-S(\rho) .
$$

The classical correlations, defined using relative entropy, are

$$
C=S\left(\chi_{\rho} \| \pi_{\chi_{\rho}}\right)=S\left(\pi_{\chi_{\rho}}\right)-S\left(\chi_{\rho}\right)
$$

where $\pi_{\chi_{\rho}}$ is the nearest classical product state to quantum-classical state $\chi_{\rho}$. In general, the total correlation $T$ is different from the sum of quantum and classical correlations $(T \neq D+C)$. This difference is the relative entropy between the closest product state $\pi_{\rho}$ and the closest product state $\pi_{\chi_{\rho}}$ to quantum-classical state $\chi_{\rho}$. It is defined by [19]

$$
L=S\left(\pi_{\rho} \| \pi_{\chi_{\rho}}\right)=S\left(\pi_{\chi_{\rho}}\right)-S\left(\pi_{\rho}\right),
$$

such that the correlations as measured by relative entropy satisfy the following remarkable additivity relation

$$
T-D-C+L=0 .
$$

As already noticed, the analytical evaluation of correlations quantifiers based on relative entropy requires optimization techniques that are in general very challenging. Also, the relative entropy is not symmetrical in its arguments and subsequently it cannot be considered as a true distance. To get

computable expressions of classical and quantum correlations in the states (10) and (15), we introduce the concept of linear relative entropy. 


\subsection{Correlation quantifiers based on linear relative entropy}

We define the linear relative entropy, for two arbitrary density matrices $\rho_{1}$ and $\rho_{2}$, as

$$
S\left(\rho_{1} \| \rho_{2}\right)=\operatorname{Tr} \rho_{1}\left(\rho_{1}-\rho_{2}\right)
$$

from which we introduce the following symmetric and antisymmetric quantities

$$
S_{+}\left(\rho_{1} \| \rho_{2}\right)=S\left(\rho_{1} \| \rho_{2}\right)+S\left(\rho_{2} \| \rho_{1}\right),
$$

and

$$
S_{-}\left(\rho_{1} \| \rho_{2}\right)=S\left(\rho_{1} \| \rho_{2}\right)-S\left(\rho_{2} \| \rho_{1}\right) \text {. }
$$

The antisymmetric part (26) of the linear relative entropy writes as the difference of the linear entropies (or purities) of the states $\rho_{1}$ and $\rho_{2}$. It is given by

$$
S_{-}\left(\rho_{1} \| \rho_{2}\right)=S_{2}\left(\rho_{2}\right)-S_{2}\left(\rho_{1}\right)
$$

in terms of the linear entropy defined by

$$
S_{2}(\rho)=1-\operatorname{Tr} \rho^{2}
$$

The symmetric part (25) rewrites as

$$
S_{+}\left(\rho_{1} \| \rho_{2}\right)=\left\|\rho_{1}-\rho_{2}\right\|^{2},
$$

that is exactly the Hilbert-Schmidt distance usually used as geometric measure of quantum correlations. We notice that the relative entropy (17) and the linear relative entropy (24) coincide only for states close to a completely mixed state. Indeed, in approximating $\log \rho$ by $\rho-\mathbb{I}$ where $\mathbb{I}$ the identity matrix, the von Neumann entropy reduces to linear entropy and the relative entropy is approximated by (24). For two-qubit states $\rho$ and $\rho^{\prime}$, expressed in Fano-Bloch representation, the Hilbert-Schmidt distance (29) is

$$
S_{+}\left(\rho \| \rho^{\prime}\right)=\frac{1}{4} \sum_{\alpha, \beta}\left(R_{\alpha \beta}-R_{\alpha \beta}^{\prime}\right)^{2},
$$

where $\alpha, \beta=0,1,2,3$. The linear variant of total correlation $T$ (18), quantum correlation $D$ (20), classical correlation $C$ (21) and the quantity $L$ (22) are respectively defined by

$$
T_{2}=S_{-}\left(\rho \| \pi_{\rho}\right), \quad D_{2}=S_{-}\left(\rho \| \chi_{\rho}\right), \quad C_{2}=S_{-}\left(\chi_{\rho} \| \pi_{\chi_{\rho}}\right), \quad L_{2}=S_{-}\left(\pi_{\rho} \| \pi_{\chi_{\rho}}\right) .
$$

Using (27), it is easy to check that they satisfy the additivity relation

$$
T_{2}-D_{2}-C_{2}+L_{2}=0
$$

to be compared with (23). In the next section, we shall derive the analytical expressions of the different correlations in the states (10) and (15). 


\section{Correlations in bipartite pure states}

Having introduced the linear relative entropy (27), we now determine the pairwise correlations in the states of type (10) corresponding to the pure partitioning scheme of the $n$-qubit state (2). A special attention is also devoted to the explicit forms of its closest product, quantum-classical and quantum-classical product states.

\subsection{Total correlation}

To evaluate the explicit form of total correlation $T_{2}$ (31), we first determine the closest product state from the pure state (10). An arbitrary product state $\pi_{\rho_{k, n-k}}=\varrho_{k} \otimes \varrho_{n-k}$ writes

$$
\pi_{\rho_{k, n-k}}=\frac{1}{4}\left[\sigma_{0} \otimes \sigma_{0}+\sum_{i}^{3}\left(a_{i} \sigma_{i} \otimes \sigma_{0}+b_{i} \sigma_{0} \otimes \sigma_{i}\right)+\sum_{i, j=1}^{3} a_{i} b_{j} \sigma_{i} \otimes \sigma_{j}\right],
$$

where $\left(a_{1}, a_{2}, a_{3}\right)$ and $\left(b_{1}, b_{2}, b_{3}\right)$ stand for the Bloch vectors of the operators $\varrho_{k}$ and $\varrho_{n-k}$ respectively. We note that the density matrix $\rho_{k, n-k}$ (10) is invariant under exchange and parity symmetries. This implies that the closest product state must be also invariant under these two operations. This simplify considerably the minimization of the distance, defined by (30), between $\rho_{k, n-k}$ and $\pi_{\rho_{k, n-k}}$. Indeed, the exchange symmetry of the state $\pi_{\rho_{k, n-k}}$ imposes

$$
a_{i}=b_{i}, \quad i=1,2,3 .
$$

Also, the parity invariance of the density matrix $\left(\left[\pi_{\rho_{k, n-k}}, \sigma_{3} \otimes \sigma_{3}\right]=0\right)$ gives

$$
a_{i}=b_{i}=0, \quad i=1,2 .
$$

It follows that the distance (30), separating the states $\rho_{k, n-k}$ and $\pi_{\rho_{k, n-k}}$, writes

$$
S_{+}\left(\rho_{k, n-k} \| \pi_{\rho_{k, n-k}}\right)=\frac{1}{4}\left[2\left(R_{30}-a_{3}\right)^{2}+R_{11}^{2}+R_{22}^{2}+\left(R_{33}-a_{3}^{2}\right)^{2}\right] .
$$

It is simple to check that the minimal distance is obtained for

$$
a_{3}^{3}=R_{30} \quad \Longleftrightarrow \quad a_{3}=\sqrt[3]{\lambda_{+}-\lambda_{-}} .
$$

Explicitly, we have

$$
a_{3}=\sqrt[3]{\frac{s^{k}+s^{n-k}}{1+s^{n}}}
$$

in terms of the overlap parameter $s$. Thus, the nearest product state to the pure state $\rho_{k, n-k}$ is

$$
\pi_{\rho_{k, n-k}}=\frac{1}{4}\left[\sigma_{0} \otimes \sigma_{0}+a_{3} \sigma_{3} \otimes \sigma_{0}+a_{3} \sigma_{0} \otimes \sigma_{3}+a_{3}^{2} \sigma_{3} \otimes \sigma_{3}\right],
$$

and the total correlation is given by

$$
T_{2}\left(\rho_{k, n-k}\right)=\frac{1}{4}\left[2\left(R_{03}^{2}-a_{3}^{2}\right)+R_{11}^{2}+R_{22}^{2}+\left(R_{33}^{2}-a_{3}^{4}\right)\right],
$$

where the correlation matrix elements and the quantity $a_{3}$ are respectively given by (12) and (36). 


\subsection{Quantum correlation}

When the measurement is taken on the subsystem containing $k$ qubits, the quantum-classical state $\chi_{\rho_{k, n-k}}$ has the form (19) [12]. In this case, one can show that $\operatorname{Tr} \rho \chi=\operatorname{Tr} \chi^{2}$. This implies that the quantum correlation defined in (31) coincides with the Hilbert-Schmidt distance between the states $\rho_{k, n-k}$ and $\chi_{\rho_{k, n-k}}$. In other words, the quantum correlation $D_{2}$ based on linear relative entropy coincides with the geometric quantum discord introduced in [12. Subsequently, following the standard method to derive the geometric measure of quantum discord for a two-qubit system [12], one gets

$$
D_{2}=\frac{1}{4}\left(\lambda_{1}+\lambda_{2}+\lambda_{3}-\lambda_{\max }\right),
$$

where $\lambda_{\max }=\max \left(\lambda_{1}, \lambda_{2}, \lambda_{3}\right)$ is the largest eigenvalue of the matrix $K$ defined by

$$
K:=x x^{T}+R R^{T},
$$

where $x=\left(x_{1}=R_{03}, x_{2}=0, x_{3}=0\right)^{T}, R$ is the matrix with elements $R_{i j}(i, j=1,2,3)$. For the bipartite pure state (10), the eigenvalues of the matrix $K$ are

$$
\lambda_{1}=\lambda_{2}=4 \lambda_{+} \lambda_{-} \quad \lambda_{3}=2\left(\lambda_{+}^{2}+\lambda_{-}^{2}\right)
$$

where $\lambda_{ \pm}$are given by (9). Clearly, $\lambda_{3}$ is the largest eigenvalue of the matrix $K$ and the quantum correlation takes the simple form

$$
D_{2}\left(\rho_{k, n-k}\right)=\frac{1}{4}\left(\lambda_{1}+\lambda_{2}\right)=2 \lambda_{+} \lambda_{-} .
$$

To find the explicit expression of the closest quantum-classical state to $\rho_{k, n-k}$, we follow the procedure reported in [12] for an arbitrary two qubit system. By a straightforward calculation, one obtains

$$
\chi_{\rho_{k, n-k}}=\frac{1}{4}\left[\sigma_{0} \otimes \sigma_{0}+R_{30} \sigma_{3} \otimes \sigma_{0}+R_{30} \sigma_{0} \otimes \sigma_{3}+R_{33} \sigma_{3} \otimes \sigma_{3}\right] .
$$

\subsection{Classical correlations}

The quantum-classical state $\chi_{\rho_{k, n-k}}$ (42) possesses parity and exchange symmetries. Thus, its closest product can be derived by reproducing the method used to find the product state $\pi_{\rho_{k, n-k}}$ given by (37). This yields

$$
\pi_{\chi_{\rho_{k, n-k}}}=\frac{1}{4}\left[\sigma_{0} \otimes \sigma_{0}+a_{3} \sigma_{3} \otimes \sigma_{0}+a_{3} \sigma_{0} \otimes \sigma_{3}+a_{3}^{2} \sigma_{3} \otimes \sigma_{3}\right],
$$

which coincides with $\pi_{\rho_{k, n-k}}$ (37). Reporting the expressions of quantum-classical state $\chi_{\rho_{k, n-k}}$ (42) and its closest product state $\pi_{\chi_{\rho_{k, n-k}}}$ (43) in (31), the classical correlation takes the form

$$
C_{2}\left(\rho_{k, n-k}\right)=\frac{1}{4}\left[2\left(R_{03}^{2}-a_{3}^{2}\right)+\left(R_{33}^{2}-a_{3}^{4}\right)\right] .
$$

Finally, since the product of the quantum-classical state $\pi_{\chi_{\rho_{k, n-k}}}$ coincides with the product state $\pi_{\rho_{k, n-k}}$, one has

$$
L_{2}\left(\rho_{k, n-k}\right)=0,
$$

and using the results (38), (41), (44) and (45), one shows that the additivity relation (32) is satisfied as expected. 


\section{$5 \quad$ Correlations in bipartite mixed states}

We now come to the second partitioning scheme. In order to evaluate the total correlation in the state (15), we determine its closest state product. The explicit expressions of the nearest quantum-classical states to the state (15) are needed to derive the quantum correlation contained in the state (15). Finally, the computation of the classical correlation in (15) requires the explicit forms of the closest product states to the quantum-classical state.

\subsection{Total correlation}

To obtain the nearest product state from the two-qubit state $\rho_{12}$ (15), we write the product state as $\pi_{\rho_{12}}=\rho_{1} \otimes \rho_{2}$. We note that, since the bipartite state $\rho_{12}$ is invariant by exchanging the roles of the qubits 1 and 2, the reduced operators $\rho_{1}$ and $\rho_{2}$ must be identical. Furthermore, the density matrix (15) commutes with the operator $\sigma_{3} \otimes \sigma_{3}$ (parity invariance). It follows that the product state $\pi_{\rho_{12}}$ is necessarily of the form

$$
\pi_{\rho_{12}}=\rho_{1} \otimes \rho_{2}=\frac{1}{4}\left[\sigma_{0} \otimes \sigma_{0}+c_{3} \sigma_{3} \otimes \sigma_{0}+c_{3} \sigma_{0} \otimes \sigma_{3}+c_{3}^{2} \sigma_{3} \otimes \sigma_{3}\right] .
$$

where $c_{3} \in \mathbb{R}$. Using (25), the distance between the states $\rho_{12}$ and $\pi_{\rho_{12}}$ is

$$
S_{+}\left(\rho_{12}, \pi_{\rho_{12}}\right)=\frac{1}{4}\left[2\left(\mathcal{R}_{30}-c_{3}\right)^{2}+\mathcal{R}_{11}^{2}+\mathcal{R}_{22}^{2}+\left(\mathcal{R}_{33}-c_{3}^{2}\right)^{2}\right]
$$

which is a function of the parameter $c_{3}$. The minimal distance is obtained by setting at zero the derivative of the distance $S_{+}\left(\rho_{12}, \pi_{\rho_{12}}\right)$. This gives the following equation

$$
c_{3}^{3}+c_{3}\left(1-\mathcal{R}_{33}\right)-\mathcal{R}_{30}=0
$$

By Cardano's formula, the unique real root of this cubic equation is

$$
c_{3}=\sqrt[3]{\frac{\sqrt{\Delta}+\mathcal{R}_{30}}{2}}-\sqrt[3]{\frac{\sqrt{\Delta}-\mathcal{R}_{30}}{2}},
$$

where

$$
\Delta=\mathcal{R}_{30}^{2}+\frac{4}{27}\left(1-\mathcal{R}_{33}\right)^{3},
$$

is non negative $\left(\mathcal{R}_{33} \leq 1\right)$. Combining the definitions (26) and (31), the total correlation in the state (15) , as measured by linear relative entropy, writes

$$
T_{2}\left(\rho_{12}\right)=\frac{1}{4}\left[2\left(\mathcal{R}_{03}^{2}-c_{3}^{2}\right)+\mathcal{R}_{11}^{2}+\mathcal{R}_{22}^{2}+\left(\mathcal{R}_{33}^{2}-c_{3}^{4}\right)\right]
$$

where the correlation matrix elements are given by (16) and the quantity $c_{3}$ (49) can be re-equated in terms of the overlapping parameter $s$ so that the total correlation is a function of the variable $s$. 


\subsection{Quantum correlation}

For a measurement performed on the first qubit of $\rho_{12}$ (15), the general form of quantum-classical state is given by (19). As in the pure partitioning scheme, one verifies that the quantum correlation $D_{2}\left(\rho_{12}\right)$ coincides with the geometric measure of quantum discord $D_{\mathrm{g}}\left(\rho_{12}\right)$ defined as the HilbertSchmidt distance between the state $\rho_{12}$ and its closest quantum-classical states. Hence, using the general method proposed in [12] (see also [20] for more details), it is easy to obtain

$$
D_{2}\left(\rho_{12}\right) \equiv D_{\mathrm{g}}\left(\rho_{12}\right)=\frac{1}{4} \min \left\{\lambda_{1}+\lambda_{2}, \lambda_{2}+\lambda_{3}\right\}
$$

where

$$
\begin{gathered}
\lambda_{1}=\mathcal{R}_{11}^{2}=4 \mathcal{N}^{4}\left(1-s^{2}\right)^{2}, \\
\lambda_{2}=\mathcal{R}_{22}^{2}=4 \mathcal{N}^{4} s^{2(n-2)}\left(1-s^{2}\right)^{2}, \\
\lambda_{3}=\mathcal{R}_{03}^{2}+\mathcal{R}_{33}^{2}=4 \mathcal{N}^{4}\left[\left(1+s^{2}\right)\left(s^{2}+s^{2(n-2)}\right)+4 s^{n}\right] .
\end{gathered}
$$

To write the explicit expression of the closest quantum-classical state to $\rho_{12}$, we exploit the optimization procedure developed in [12]. For this end, we discuss separately the situations where $\lambda_{1} \leq \lambda_{3}$ and $\lambda_{3} \leq \lambda_{1}$. The condition $\lambda_{1} \leq \lambda_{3}$ writes as

$$
\left(s^{2}+1\right)\left(1+s^{n-2}\right)-2\left(1-s^{2}\right) \geq 0
$$

In this case, the closest quantum-classical state is obtained as

$$
\chi_{\rho_{12}}^{-}=\frac{1}{4}\left[\sigma_{0} \otimes \sigma_{0}+\mathcal{R}_{30} \sigma_{3} \otimes \sigma_{0}+\mathcal{R}_{30} \sigma_{0} \otimes \sigma_{3}+\mathcal{R}_{33} \sigma_{3} \otimes \sigma_{3}\right],
$$

where the subscript ${ }^{-}$refers to the situation where the difference $\lambda_{1}-\lambda_{3}$ is non positive. It follows that the quantum correlation present in the two qubit state $\rho_{12}$ is

$$
D_{2}^{-}\left(\rho_{12}\right)=S_{-}\left(\rho_{12} \| \chi_{\rho_{12}}^{-}\right)=\frac{1}{4}\left(\lambda_{1}+\lambda_{2}\right)=\frac{1}{4}\left(\mathcal{R}_{11}^{2}+\mathcal{R}_{22}^{2}\right),
$$

which rewrites as

$$
D_{2}^{-}\left(\rho_{12}\right)=D_{\mathrm{g}}^{-}\left(\rho_{12}\right)=\frac{1}{4} \frac{\left(1+s^{2(n-2)}\right)\left(1-s^{2}\right)^{2}}{\left(1+s^{n}\right)^{2}} .
$$

The second class of closest quantum-classical states is obtained in the case where $\lambda_{1}-\lambda_{3} \geq 0$ corresponding to the situation when the condition

$$
\left(s^{2}+1\right)\left(1+s^{n-2}\right)-2\left(1-s^{2}\right) \leq 0
$$

is satisfied. In this case, the closest quantum-classical states take the form

$$
\chi_{\rho_{12}}^{+}=\frac{1}{4}\left[\sigma_{0} \otimes \sigma_{0}+\mathcal{R}_{03} \sigma_{0} \otimes \sigma_{3}+\mathcal{R}_{11} \sigma_{1} \otimes \sigma_{1}\right],
$$

where now the subscript + refers to the sign of the difference $\lambda_{1}-\lambda_{3}$. It follows that the quantum correlation is

$$
D_{2}^{+}\left(\rho_{12}\right)=S_{-}\left(\rho \| \chi_{\rho}^{+}\right)=\frac{1}{4}\left(\lambda_{2}+\lambda_{3}\right)=\frac{1}{4}\left(\mathcal{R}_{22}^{2}+\mathcal{R}_{03}^{2}+\mathcal{R}_{33}^{2}\right)
$$


Explicitly, it is given by

$$
D_{2}^{+}\left(\rho_{12}\right)=D_{\mathrm{g}}^{+}\left(\rho_{12}\right)=\frac{1}{4} \frac{\left(s^{2}+s^{2(n-2)}\right)\left(1+s^{2}\right)+4 s^{n}+s^{2(n-2)}\left(1-s^{2}\right)^{2}}{\left(1+s^{n}\right)^{2}} .
$$

From equations (56) and (60), it is simple to check that the closest quantum-classical states $\chi_{\rho_{12}}^{ \pm}$ satisfy the relation

$$
\operatorname{Tr} \rho_{12} \chi_{\rho_{12}}^{ \pm}=\operatorname{Tr}\left(\chi_{\rho_{12}}^{ \pm}\right)^{2}
$$

which implies that the geometric discord $D_{\mathrm{g}}\left(\rho_{12}\right)$, as measured by Hilbert-Schmidt norm, coincides indeed with quantum correlation $D_{2}\left(\rho_{12}\right)$ defined by means of linear relative entropy.

\subsection{Classical correlations}

To evaluate the classical correlation defined by (31), one has to determine first the closest product

states to quantum-classical states $\chi_{\rho_{12}}^{ \pm}$given by (56) and (60). Let us denote them by $\pi_{\chi_{\rho_{12}}^{ \pm}}$. To derive the product state $\pi_{\chi_{\rho_{12}^{-}}^{-}}$, we notice that the permutation and parity symmetries leave the state $\chi_{\rho_{12}}^{-}$ (56) invariant. Therefore, reiterating the method discussed above in obtaining the product states $\left(\pi_{\rho_{12}}\right.$ for instance), it is simple to check that the closest product state to quantum-classical state $\chi_{\rho_{12}}^{-}(56)$ is

$$
\pi_{\chi_{\rho_{12}}}=\frac{1}{4}\left[\sigma_{0} \otimes \sigma_{0}+c_{3} \sigma_{3} \otimes \sigma_{0}+c_{3} \sigma_{0} \otimes \sigma_{3}+c_{3}^{2} \sigma_{3} \otimes \sigma_{3}\right]
$$

which coincides with the product state $\pi_{\rho_{12}}$. The classical correlation (31) is then given by

$$
C_{2}^{-}\left(\rho_{12}\right)=\frac{1}{4}\left[2\left(\mathcal{R}_{03}^{2}-c_{3}^{2}\right)+\left(\mathcal{R}_{33}^{2}-c_{3}^{4}\right)\right]
$$

For the state $\chi_{\rho_{12}}^{+}$, the situation is slightly different. This state is not invariant under the permutation of the qubits 1 and 2. Only the parity symmetry is preserved. The general form of the closest classical product states, symmetric under parity transformation, is

$$
\pi_{\chi_{\rho_{12}}}^{+}=\frac{1}{4}\left[\sigma_{0} \otimes \sigma_{0}+\alpha_{3} \sigma_{3} \otimes \sigma_{0}+\beta_{3} \sigma_{0} \otimes \sigma_{3}+\alpha_{3} \beta_{3} \sigma_{3} \otimes \sigma_{3}\right],
$$

where $\alpha_{3}$ and $\beta_{3}$ stand for the variables to be optimized to get the minimal Hilbert-Schmidt distance between the states $\chi_{\rho_{12}}^{+}$and $\pi_{\chi_{\rho_{12}}^{+}}$. A simple calculation leads to

$$
\alpha_{3}=0, \quad \beta_{3}=\mathcal{R}_{03} .
$$

Then, the closest product state writes

$$
\pi_{\chi_{\rho_{12}}^{+}}=\frac{1}{4}\left[\sigma_{0} \otimes \sigma_{0}+\mathcal{R}_{03} \sigma_{0} \otimes \sigma_{3}\right],
$$

and the classical correlation reads

$$
C_{2}^{+}\left(\rho_{12}\right)=\frac{1}{4} \mathcal{R}_{11}^{2} \text {. }
$$


Using the expression of the product state $\pi_{\rho_{12}}$ (46) and the classical product states $\pi_{\chi_{\rho_{12}}^{-}}$(63) and $\pi_{\chi_{\rho_{12}}^{+}}$(66) , the expression of the quantity $L_{2}$ (31) is given by

$$
L_{2}^{-}\left(\rho_{12}\right)=0, \quad L_{2}^{+}\left(\rho_{12}\right)=\frac{1}{4}\left[2 c_{3}^{2}+c_{3}^{4}-\mathcal{R}_{03}^{2}\right]
$$

which satisfy

$$
L_{2}^{ \pm}=D_{2}^{ \pm}+C_{2}^{ \pm}-T_{2}
$$

and one has the additivity relation (32). The analysis presented in this section show clearly the advantages of the linear relative entropy (24) in deriving the expressions of the different correlations explicitly in terms of the overlapping parameter $s$ and the number $n$ of qubits in the state (2). This is essentially due to the simplification arising in determining the product, quantum-classical and quantum-classical product states for the bipartite states $\rho_{12}$. To analyze the behavior of the obtained correlations as functions of $n$ and the parameter $s$, we shall discuss some specific cases.

\section{Illustration: some special cases}

As illustration, we consider some special instances of the states (2). We shall especially give the analytical expressions of the total, quantum and classical pairwise correlations for $n=2$ and $n=3$. Numerical results for states containing more qubits are also discussed.

\subsection{Two qubits}

For $n=2$, the total correlation (38) writes

$$
T_{2}\left(\rho_{1,1}\right)=\frac{1}{4}\left[\left(1-2\left(\frac{2 s}{1+s^{2}}\right)^{\frac{2}{3}}\right)\left(1-\left(\frac{2 s}{1+s^{2}}\right)^{\frac{4}{3}}\right)+2\left(\frac{1-s^{2}}{1+s^{2}}\right)^{2}\right] .
$$

From equation (41), the quantum correlation reads

$$
D_{2}\left(\rho_{1,1}\right)=\frac{1}{2}\left(\frac{1-s^{2}}{1+s^{2}}\right)^{2},
$$

and using (44), the classical correlation is given by

$$
C_{2}\left(\rho_{1,1}\right)=\frac{1}{4}\left(1-2\left(\frac{2 s}{1+s^{2}}\right)^{\frac{2}{3}}\right)\left(1-\left(\frac{2 s}{1+s^{2}}\right)^{\frac{4}{3}}\right) .
$$

Clearly, we have the additivity relation

$$
T_{2}\left(\rho_{1,1}\right)=D_{2}\left(\rho_{1,1}\right)+C_{2}\left(\rho_{1,1}\right) .
$$

The quantity $L_{2}$ (45) is zero for pure states. These results can be equivalently obtained from ones derived for the second bipartite partitioning scheme. Indeed, for $n=2$ the state $\rho_{12}$ (14) is pure. Also, it is simple to verify that for $n=2$ the solutions (35) and (49) are identical. It follows that, for 
$n=2$, total correlation $T_{2}\left(\rho_{1,1}\right)$ and $T_{2}\left(\rho_{12}\right)$ coincide. Moreover, the condition (55) is always satisfied for $n=2$. This implies that the quantum correlation is given by

$$
D_{2}\left(\rho_{12}\right) \equiv D_{2}^{-}\left(\rho_{1,1}\right)=\frac{1}{2}\left(\frac{1-s^{2}}{1+s^{2}}\right)^{2},
$$

which is exactly the quantum discord (171). Similarly, using the fact that the solutions (35) and (49) are identical for $n=2$, one has

$$
C_{2}\left(\rho_{12}\right)=C_{2}\left(\rho_{1,1}\right)
$$

\subsection{Three qubits}

The situation is slightly different for $n=3$. We shall focus only on the mixed partitioning scheme. In this case, the correlation matrix elements (16) read

$$
\mathcal{R}_{00}=1, \quad \mathcal{R}_{11}=\frac{1-s^{2}}{1+s^{3}}, \quad \mathcal{R}_{22}=-s \frac{1-s^{2}}{1+s^{3}}, \mathcal{R}_{33}=\frac{s+s^{2}}{1+s^{3}}, \quad \mathcal{R}_{03}=\mathcal{R}_{30}=\frac{s+s^{2}}{1+s^{3}}
$$

Reporting $\mathcal{R}_{03}$ and $\mathcal{R}_{33}$ in (49), one has the expression of $c_{3}$ (49) and subsequently the total correlation (50) writes as a function of the overlap $s$. The behavior of $T_{2}$ versus $s$ is given in figure 1 . The condition

(55) (resp. (59)) is satisfied when $\sqrt{2}-1 \leq s \leq 1$ (resp. $0 \leq s \leq \sqrt{2}-1$ ). It follows that the quantum discord is given by

$$
D_{2}^{-}\left(\rho_{12}\right)=\frac{1}{4} \frac{\left(1-s^{2}\right)^{2}\left(1+s^{2}\right)}{\left(1+s^{3}\right)^{2}}
$$

for $\sqrt{2}-1 \leq s \leq 1$, and

$$
D_{2}^{+}\left(\rho_{12}\right)=\frac{1}{4} \frac{s^{2}(1+s)^{2}\left(2+(1-s)^{2}\right)}{\left(1+s^{3}\right)^{2}}
$$

for $0 \leq s \leq \sqrt{2}-1$. Furthermore, the classical correlation $C_{2}^{-}\left(\rho_{12}\right)\left(\right.$ resp. $\left.C_{2}^{+}\left(\rho_{12}\right)\right)$ when $\sqrt{2}-1 \leq s \leq 1$ (resp. $0 \leq s \leq \sqrt{2}-1$ ) is obtainable from the equation (64) (resp 67) by simply replacing the correlation matrix elements and $c_{3}$ by their corresponding expressions in terms of the parameter $s$ for $n=3$. Similarly, one can write the explicit form of the quantity $L_{2}^{+}\left(\rho_{12}\right)(68)$ as a function of $s$. The total pairwise correlations $T_{2}(50)$ for the states comprising three qubits or more $(n \geq 3)$ are depicted in figure 1. It is remarkable that the maximal amount of total correlation, as measured by linear relative entropy, exists in states comprising three qubits. It is interesting also to note that the total pairwise correlation in the states $\rho_{12}(14)$ becomes identical for all the states containing four qubits and more. 


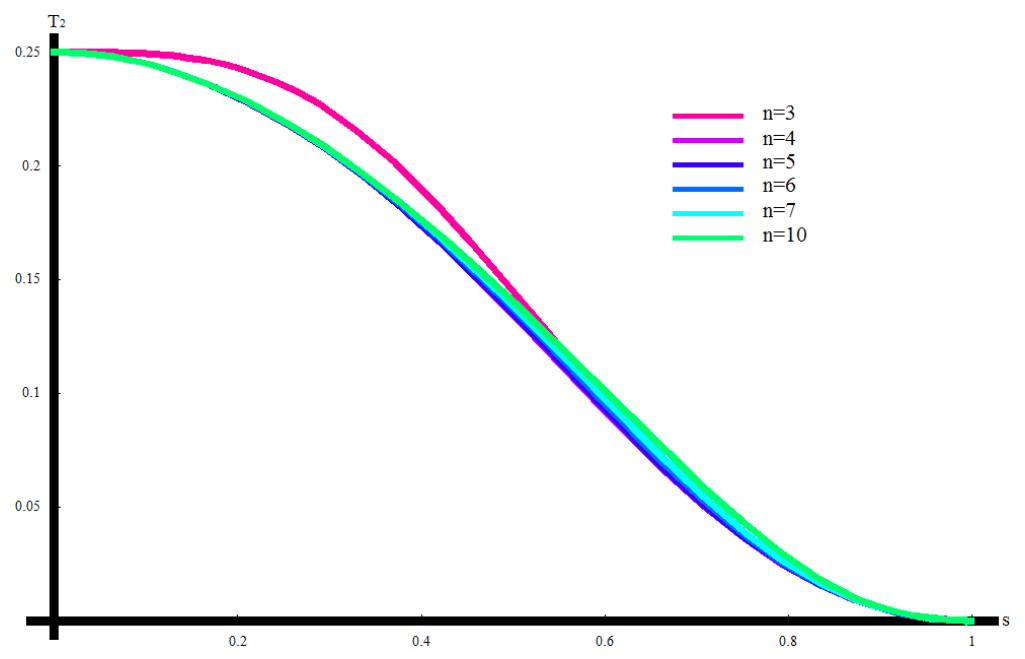

Figure 1. The total pairwise correlation $T_{2}$ versus the overlapping $s$ for different values of $n$.

We also consider the behavior of quantum discord given by the equations (58) and (62) for different values of qubits number $n$. This is reported in figure 2. The quantum discord increases first and start suddenly decreasing to vanishes for $s=1$. The sudden change of quantum discord is responsible of the violation of the additivity relation and induces the discontinuity of classical correlations as it is shown in figure 3. It is also clearly seen that the pairwise quantum correlations have approximately the same behavior for states with $n \geq 4$. This is similar to what happens with total correlation (see figure 1). The figure 3 presenting the variation of classical correlation versus the overlap parameter $s$ shows two important features. First, like total and quantum correlations in states with $n \geq 4$, the classical correlation becomes independent of $n$. The second important feature is that the pairwise classical correlation, as measured by linear relative entropy, is discontinuous when crossing the point where the quantum discord suddenly changes (see figure 2). This reflects the fact that the deviation from the additivity relation $\left(T_{2} \neq D_{2}^{+}+C_{2}^{+}\right)$and the discontinuity of classical correlation are deeply related to the sudden change of quantum discord. To study the violation of the additivity relation, we reported in figure 4 , the difference $L_{2}$ between total correlation $T_{2}$ and the sum of quantum and classical correlation $\left(D_{2}+C_{2}\right)$. For states with $n \geq 5$, the behavior of $L_{2}$ versus $s$ becomes slightly identical when $n$ increases (figure 4 ). The violation of the additivity relation is more prominent for states containing small number of qubits. 


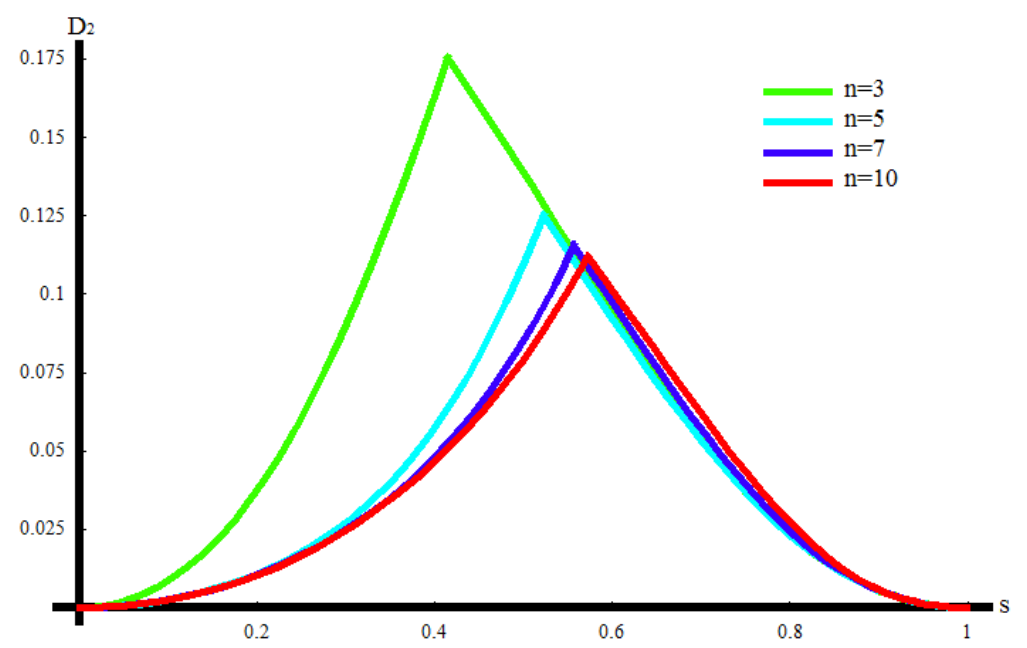

Figure 2. The quantum discord $D_{2}$ versus the overlapping $s$ for different values of $n$.

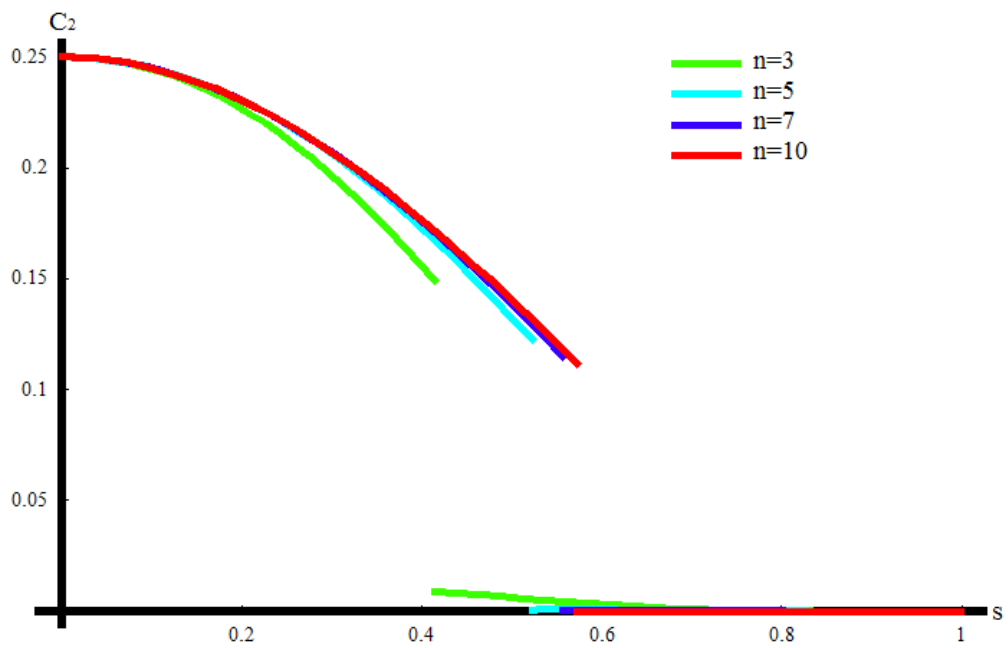

Figure 3. The classical correlation $C_{2}$ versus the overlapping $s$ for different values of $n$.

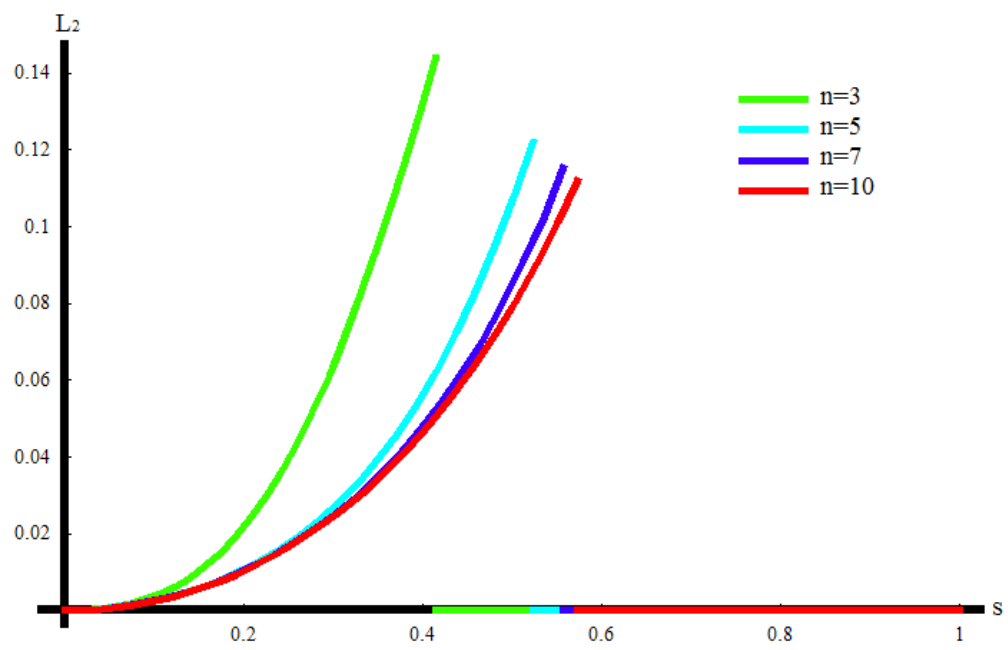

Figure 4. The difference $L_{2}=D_{2}+C_{2}-T_{2}$ versus the overlapping $s$ for different values of $n$. 


\section{Concluding remarks}

In this paper, we have obtained explicit expressions for total, classical and quantum pairwise correlations using the linear relative entropy. This variant of relative entropy, though restricted from an informational point of view, constitutes an advantageous tool to get analytically computable quantities. Furthermore, it allows us to deal with the different kinds of correlations in a common framework. This is reflected by the closed additivity relation among the pairwise correlations. We deliberately considered a $n$ qubit system, prepared in states of the form (2), to emphasize the usefulness of linear relative entropy in quantifying the correlations in a unified way. To evaluate the amount of the various pairwise correlations, two different bi-partitioning schemes were examined. The first scheme deals with pure states and the second, obtained by a trace procedure, concerns bipartite mixed states. For bipartite pure states, the total correlation coincides with the sum of classical and quantum cor-

relations. However, in bipartite mixed states, this relation is not always valid: $T_{2} \neq D_{2}^{+}+C_{2}^{+}$(see equation (69)). The non-vanishing quantity $L_{2}^{+}$given by (68), measuring the relative entropy between the product and product quantum-classical states, is responsible of this inequality. At this stage, it is interesting to note that the sudden change of the quantum discord, when crossing the transition

point $\lambda_{1}=\lambda_{3}$ (cf. (55) and (59) ), is accompanied by the apparition of the non-vanishing quantity $L_{2}^{+}$ which diminishes the sum of classical and quantum correlations in comparison with total correlation. Finally, we notice that the obtained pairwise correlations are useful to capture the essential of genuine multipartite correlation present in the state (2). The global multipartite correlation, defined as the sum of all possible pairwise correlations in a multi-qubit system (see for instance [24, 25]), can be evaluated using the results derived here through the formalism of linear relative entropy. We hope to report in this issue in a forthcoming work.

\section{References}

[1] R. Horodecki, P. Horodecki, M. Horodecki and K. Horodecki, Rev. Mod. Phys. 81(2009) 865.

[2] O. Gühne and G. Tóth, Phys. Rep. 474 (2009) 1.

[3] M.A. Nielsen and I.L. Chuang, Quantum Computation and Quantum Information (Cambridge Univ. Press, Cambridge, 2000).

[4] V. Vedral, Rev. Mod. Phys. 74 (2002) 197.

[5] K. Modi, A. Brodutch, H. Cable, T. Paterek and V. Vedral, Rev. Mod. Phys. 84 (2012) 1655.

[6] P. Rungta, V. Buzek, C.M. Caves, M. Hillery and G.J. Milburn, Phys. Rev. A 64 (2001) 042315.

[7] C.H. Bennett, D.P. DiVincenzo, J. Smolin and W.K. Wootters, Phys. Rev. A 54 (1996) 3824.

[8] W.K. Wootters, Phys. Rev. Lett. 80 (1998) 2245. 
[9] V. Coffman, J. Kundu and W.K. Wootters, Phys. Rev. A 61 (2000) 052306.

[10] L. Henderson and V. Vedral, J. Phys. A 34(2001) 6899; V. Vedral, Phys. Rev. Lett. 90 (2003) 050401; J. Maziero, L. C. Celéri, R.M. Serra and V. Vedral, Phys. Rev A 80 (2009) 044102.

[11] H. Ollivier and W.H. Zurek, Phys. Rev. Lett. 88 (2001) 017901.

[12] B. Dakic, V. Vedral and C. Brukner, phys. Rev. Lett. 105 (2010) 190502.

[13] B. Bellomo, R. Lo Franco and G. Compagno, Phys. Rev. A 86 (2012) 012312.

[14] B. Bellomo, G.L. Giorgi, F. Galve, R. Lo Franco, G. Compagno and R. Zambrini, Phys. Rev. A 85 (2012) 032104.

[15] J. Dajka, J. Luczka and P. Hänggi, Phys. Rev. A 84 (2011) 032120.

[16] J.D. Montealegre, F.M. Paula, A. Saguia and M.S. Sarandy, Phys. Rev. A 87 (2013) 042115.

[17] B. Aaronson, R. Lo Franco, G. Compagno and G. Adesso, New Journal of Physics 15 (2013) 093022 .

[18] F.M. Paula, T.R. de Oliveira and M.S. Sarandy, Phys. Rev. A 87 (2013) 064101.

[19] K. Modi, T. Paterek, W. Son, V. Vedral and M. Williamson, Phys. Rev. Lett. 104 (2010) 080501.

[20] M. Daoud and R. Ahl Laamara, Phys. Lett. A 376 (2012) 2361.

[21] M Daoud, R Ahl Laamara and W Kaydi, J. Phys. A: Math. Theor. 46 (2013) 395302.

[22] L. Henderson and V. Vedral, J. Phys. A 34 (2001) 6899; V. Vedral, Phys. Rev. Lett. 90 (2003)050401; J. Maziero, L.C. Celéri, R.M. Serra, and V. Vedral, Phys. Rev A 80 (2009) 044102.

[23] H. Ollivier and W.H. Zurek, Phys. Rev. Lett. 88 (2001) 017901.

[24] C.C. Rulli and M.S. Sarandy, Phys. Rev. A 84 (2011) 042109.

[25] Z-H Ma, Z-H Chen and F.F. Fanchini, New J. Phys. 15 (2013) 043023. 